\title{
A Lattice-Theoretical Approach to Modeling Naturally Ordered Structures
}

\author{
Olga Shabalina \\ CAD Department \\ Volgograd State Technical University \\ Volgograd, Russia \\ E-mail: o.a.shabalina@gmail.com
}

Alexander Davtian

Mathematics and Mathematical Methods in Physics

Moscow Institute of Physics and Technology

Moscow, Russia

E-mail: agvs@mail.ru

\author{
Dmitriy Yerkin \\ CAD Department \\ Volgograd State Technical University \\ Volgograd, Russia \\ E-mail: hate.cska@gmail.com
}

\author{
Natalia Sadovnikova \\ CAD Department \\ Volgograd State Technical University \\ Volgograd, Russia \\ E-mail:npsn1@ya.ru
}

\begin{abstract}
Different problems arising in system analysis and design need building system models describing their internal structure. Graph representation that is commonly used in this case allows to present system structure only as a set of related components. For reflecting specific structural properties of modeling system structural model of such system should possess additional characteristics reflecting corresponding properties. There exists a class of systems, characterized by natural structural order of its elements. The paper considers an approach of modeling systems with naturally ordered structures, including mathematical model representing the system structure, declarative markup language and programming tool for building such model.
\end{abstract}

Keywords - structural model; natural structural order; latticetheoretical approach;lattice; markup language.

\section{INTRODUCTION}

Different problems arising in system analysis and design need building system models describing their internal structure. Structural models are usually described by graphs with vertices as system components and edges representing relationship between the components. Graph theory has been well developed; there exist a lot of tools for graphs building and visualization. But graph representation reflects nothing more than a binary relation and it allows describing system structure only as a set of related components. For reflecting specific structural properties of modeling system structural model of such system should possess additional characteristics reflecting corresponding properties.

There exists a class of systems, characterized by natural structural order of its elements. Structural ordering reflects wholeness and interdependence of system structure and allows decomposing the system keeping wholeness of system fragments, while each fragment of the structure is determined

The study was financially supported by RFBR, research projects №№ 1607-00611\16a, 16-07-00353\16a by the structure itself.

The paper considers an approach of modeling systems with naturally ordered structures, including mathematical model representing the system structure, declarative markup language and programming tool for building such model.

\section{RELATED WORK}

In mathematics for representing ordering relationship partially ordered sets (posets) are used. But poset-based structural models may not always reflect wholeness and interdependence of system structure. The most common mathematical representation of such properties is lattice, a partially ordered set in which every two elements have a unique supremum and a unique infimum.

Partially ordered sets and lattices have been applied to modeling of different systems in different fields of scientific study [1].

A Lattice-Theoretical Approach (LTA) is used in Text Mining for formal representation of conceptual knowledge $[2,3]$, in sociology (in particular in social networks analysis) [3], in analysis of data of sociological surveys, etc. Concept lattice is usually represented as a matrix with rows corresponding to some objects, and columns - to attributes. Relationships between objects and attributes are indicated in the matrix cells. Specialized programing tools have been developed for building concept lattices using matrix description (Concept Explorer [4], Lattice Miner [5], etc.).

Lattice-based knowledge space model was suggested in [6] for describing educational resources for nonlinear educational games. Using such model allows to combine learning a gaming process and to manage a non-linear game scenario.

In recent year LTA has been used for technical system structure modeling. Thus, in [7] a lattice-based structure model 
is used for synthesizing different structurally implemented schemes of machine and mechanical appliance decomposition.

For describing lattice-based models in terms of graph existing markup languages are used. Most of such languages (GraphML [8], GXL [9], XGMML[10], etc.) were developed as extensions of XML and there are some alternative conceptions (e.g. GML[11]). But the use of those languages for describing a lattice-based structural model is limited to cases when the system structure is known and it is exactly a lattice. And besides any modification of initial model structure leads to the necessity to rebuild structure description.

EMMA markup language [12] is also an extension of XML but it contains special namespace for describing lattice objects. But in any case using EMMA for describing latticebased structural models requires knowing its lattice structure. Also XML-like EMMA's syntax looks very bulky as it is based on a hierarchical data model and contains a lot of tags which are not necessary for lattice markup.

\section{LATTICE-BASED STRUCTURAL MODEL}

Initial structure of any naturally ordered system can be presented as a finite poset:

$$
I S=\langle C, \leq\rangle
$$

where $S$ - structural model,

$I S=\left\{s_{1}, s_{2}, s_{3}, \ldots\right\}-$ set of system elements,

$\leq$ - binary relation over a set $S$.

The binary relation $\leq$ reflects logical conditionality and semantic coherence of system fragments. Fig.1.

An example of initial structural model is shown on the

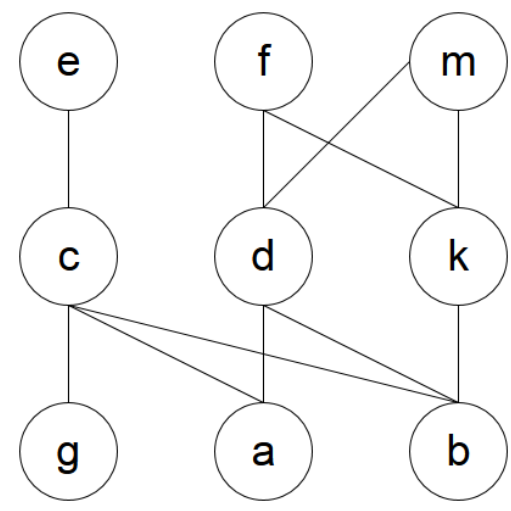

Fig. 1. Hasse diagram of initial structural model

In most cases the initial structure built by a model developer reflects the very general structural properties and thus most likely is not structurally-ordered. For reflecting this property the initial structure (1) is embedded into a lattice $F S \supset I S$ for ensuring wholeness and structural fragmentability of the model.

The final lattice-based structural model of the structure shown on the Fig. 1 is shown on the Fig. 2.

The appearance of new elements - a least upper bound (supremum) and a greatest lower bound (infimum) for each pair of elements in the final structure $F S$ reflects aggregate structural properties of the system.

For each pair $s_{1}, s_{2}$ of elements related as $s_{1} \leq s_{2}$, a set of elements $x \in F S: s_{1} \leq x \leq s_{2}$ defines the interval $I_{s_{1}}^{s_{2}}$ as a substructure of the structure $F S$. Existence of substructures $I_{s_{1}}^{s_{2}} \subset F S$ determines the structural similarity of the structural model, and reflects its divisibility.

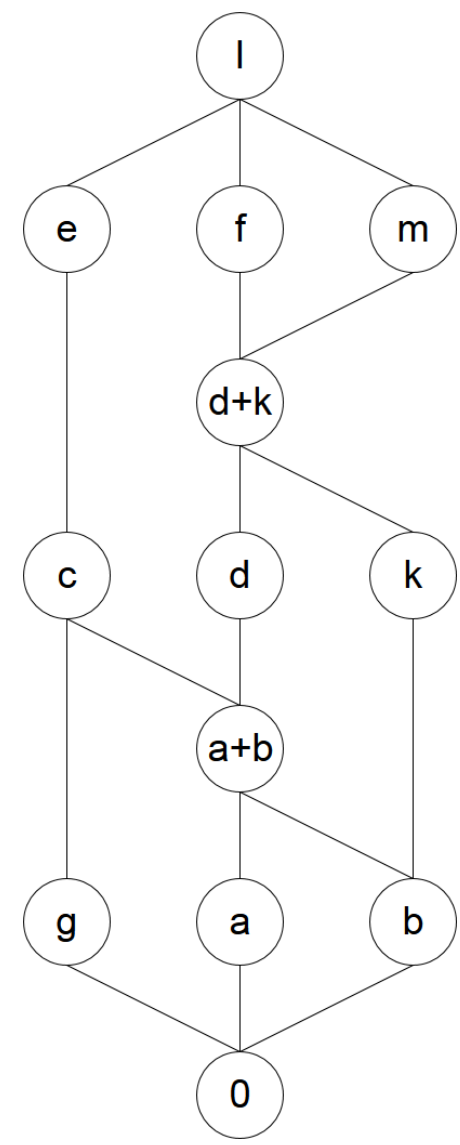

Fig. 2. Hasse diagram of final structural model

The lattice $F S$ allows existence of the intervals $I_{s_{1}}^{s_{2}}$, containing maximal chains of different length: $\left|C_{s_{1 i}}^{s_{2}} \subset I_{s_{1}}^{s_{2}}\right| \neq\left|C_{s_{1} j}^{s_{2}} \subset I_{s_{1}}^{s_{2}}\right|$, i.e., in general, the lattice can be non-modular. Non-modularity means impossibility of combining structure substructures without losing its structural equivalence i.e. reflects system structural complexity. 
Existence of intervals $I_{s_{1}}^{s_{2}}$ containing more than two maximal chains $L\left(I_{s_{1}}^{s_{2}}\right) \geq 2$, where $L-$ is a number of linear orders of interval $I_{s_{1}}^{s_{2}}$, means that, in the general case, the lattice can be non-distributive. Lattice non-distributivity means non-reducibility of the structure to a simple combination of substructures, i.e. reflects synergistic properties of structural model.

\section{MARKUP LANGUAGE FOR LATTICE-BASED STRUCTURAL MODELS DESCRIPTION}

For describing lattice-based structural models a specialized markup language has been developed. Each element of the lattice is identified by a unique name composed of Latin letters, numbers and underscore symbol. Each element can be matched to unlimited set of properties; each property is described by its name and value enclosed in square brackets.

The lattice description consist of four blocks - two mandatory blocks and two optional ones. Mandatory block are the following:

- "Source" block - for pointing lattice infimum;

- “Target” block - for pointing lattice supremum.

The optional blocks are:

- "Elements" - for pointing lattice elements;

- "Orders" - for describing the order of elements.

The order of elements is described by the ">" symbol with one greater element on the left and smaller elements on the right. If the number of smaller elements is more than one, their names must are separated by the " $\&$ " symbol.

The syntax of the language in extended Backus-Naur Form is described below:

$$
\begin{aligned}
& \text { Lattice }=\text { Source Target [Nodes] [Orders]. } \\
& \text { Source = "Source: " Element. } \\
& \text { Target }=\text { "Target: } " \text { Element. } \\
& \text { Elements = "Element: "Element, \{";" Element }\} \text { "End". } \\
& \text { Orders = "Orders:" Order, \{";" Order \} "End". } \\
& \text { Node = Name }\{\text { Property }\} \text {. } \\
& \text { Property = "[" Name "=" "'" Value "'" "]" \{Property\}. } \\
& \text { Name }=\text { Letter } \mid \text { Digit }\{\text { Letter } \mid \text { Digit } \mid "-"\} \text {. } \\
& \text { Digit = "0"| "1"| "2"| "3"| ... | "7" | "8" | "9". }
\end{aligned}
$$

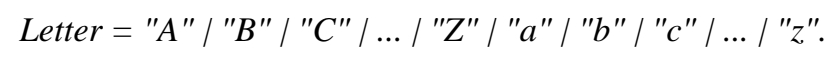

$$
\begin{aligned}
& \text { Order = Name ">" Name \{"\&" Name }\} \text { ";". }
\end{aligned}
$$

\section{PROGRAMMING TOOL FOR BUILDING STRUCTURAL MODELS}

For building structural models specialized programming system has been developed. The main function of the system is to build and visualize lattices from their description on suggested markup language.

The main window contains two frames. The left frame is intended for describing lattice-based structural models in markup language. The text editor supports syntax highlighting for keywords and main constructions of the language grammar. The right frame represents the built model visualization. The system provides drag-and-drop support and an access to lattice elements properties by clicking on them.

Developed markup language parser provides notifications for possible syntax mistakes in lattice description.

The screenshot of the main window is given on Fig. 3

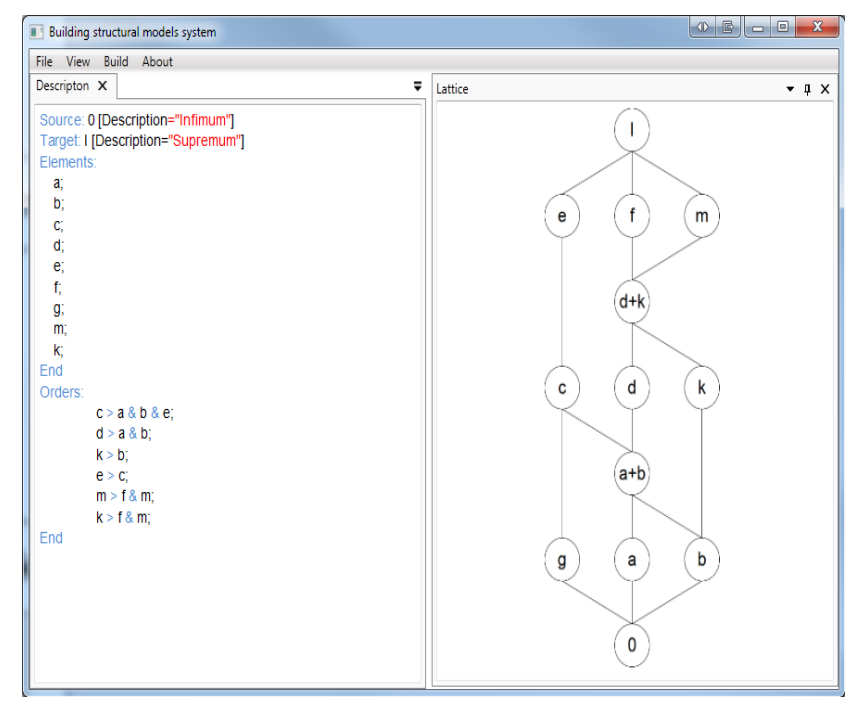

Fig. 3. Screenshot of programming system main window

\section{CONCLUSIONS}

In this paper a lattice-theoretical approach to modeling naturally structured systems is presented. Lattice-based structural models allow analyzing system structure in terms of wholeness and structural order. The paper presents a specialized markup language and programming tool for building and visualizing lattice-based structural models.

\section{REFERENCES}

[1] Davey, B. A. and Priestley, H. A Introduction to Lattices and Order. 2nd. Ed. Cambridge: Cambridge University Press, 2002.

[2] Ganter, Bernhard; Stumme, Gerd; Wille, Rudolf, eds., Formal Concept Analysis: Foundations and Applications, Lecture Notes in Artificial Intelligence, No. 3626, Springer-Verlag, 2005.

[3] Freeman, L., Cliques, Galois lattices, and the structure of human social groups, Social Networks 18, pp. 173-187 (1996).

[4] Concept Explorer [Online]. Available: http://conexp.sourceforge.net/download.html.

[5] Lattice Miner [Online]. Available: http://lattice-miner.soft112.com/

[6] Shabalina O., Vorobkalov P. Development of Educational Computer Games: Learning Process Model and how it is Integrated into the Game Context. World Applied Sciences Journal (WASJ). 2013. Vol. 24, Spec. Issue 24 : Information Technologies in Modern Industry, Education \& Society, pp. 256-267 
[7] Bojko A., N. Theoretically-lattice model of machine and mechanical device decomposition [Online]. Available: http://technomag.bmstu.ru/doc/324631.html

[8] The GraphML File Format [Online]. Available: http://graphml.graphdrawing.org/

[9] GXL - Graph eXchange Language [Online]. Available: http://www.gupro.de/GXL/

[10] XGMML (eXtensible Graph Markup and Modeling Language) [Online] Available:

http://cgi7.cs.rpi.edu/research/groups/pb/punin/public_html/XGMML/
[11] GML: A portable Graph File Format [Online]. Available: http://www.fim.uni-passau.de/fileadmin/files/lehrstuhl/

brandenburg/projekte/gml/gml-technical-report.pdf

[12] EMMA: Extensible MultiModal Annotation markup Language [Online]. Available: http://www.w3.org/TR/emma/

[13] Yerkin D., Shabalina O. Razrabotka biblioteki postroeniya strukturnykh modeley sistem. Izvestiya Volgogradskogo gosudarstvennogo tekhnicheskogo universiteta. 2015. № 13 (177), pp. 61-65. 